\title{
Ist die Reliefenergie ein Maß für das Alter der Endmoränen?
}

\author{
Von Gerd Lüttig, Hannover \\ Mit 2 Abbildungen und 1 Tabelle
}

I $\mathrm{n}$ h a $1 \mathrm{t}$. Versucht wird, die bisher mit Hilfe der morphologischen Eigenart von Endmoränen meist subjektiv geführte Beweisführung für das Alter von Moränen auf eine durch Messung nachprüfbare Basis zu stellen. Die Reliefenergiekarte bildet dazu ein ausgezeichnetes Hilfsmittel.

$\mathrm{Abstract}$. The attempt is made to put the process of proving the age of moraines which hitherto has mostly been done in a subjective manner with the help of the morphological properties of end moraines, on a basis to be verifid by measurements. For this purpose an excellent aid is given by the relief energy map.

0.

In geologischen und geomorphologischen Arbeiten, die über Endmoränen handeln, findet man häufig Vermerke, aus denen hervorgeht, daß man der Form einer Endmoräne eine Aussagekraft im Hinblick auf das Alter zumißt. Entweder wird gesagt, daß eine Moräne eine verwaschene Form zeige, weshalb sie besonders alt sein soll, oder besonders frisch aussehe, weswegen mit einem geringen Alter der Endmoräne gerechnet werden müsse. Es gibt auch Fälle, in denen die Behauptung aufgestellt wird, eine Moräne sei relativ alt, weil sie - im Vergleich zu jüngeren Moränen - ein durch jüngere Unterschneidung belebtes Relief besitzt. Kurzum: Die Form der Endmoräne hat - bewußt oder unbewußt - bei der Alterseinstufung eine mehr oder minder große Rolle gespielt.

Bisher ist noch kein Versuch unternommen worden, die sehr subjektiven und oftmals suggestiven Äußerungen durch wenigstens annähernd exakte Daten zu untermauern.

1.

Mit Hilfe der Reliefenergiekarte ist es möglich, dieser Frage etwas präziser nachzugehen. Bei den folgenden Untersuchungen ist die Methode des Verfassers (LÜTTIG 1953, 1955) verwandt worden. Damit soll nicht unterstellt werden, daß die Methode vor anderen (z. B. den von Waldbauer 1952, Behrens 1953 und Thauer 1955 entwickelten) prinzipiell vorgezogen werden muß.

Die Kreismethode verwendet das Gefälle oder den Steigungsgrad (in \%) als Ausdruck der Reliefenergie. Die Karten enthalten Linien gleichen Steigungsgrades mit folgenden Größen: $1 \%, 2,5 \%, 5 \%, 7,5 \%, 10 \%, 20 \%$. Im vorliegenden Falle sind die Höhenschichtlinien im 10-m-Abstand abgegriffen worden. Das erwies sich als zweckmäßigste Abstufung. $\left.{ }^{1}\right)$ Die Flächen zwischen den Linien gleichen Steigungsgrades wurden farbig angelegt, der durchschnittliche Steigungsgrad sodann graphisch ermittelt.

Um Irrtümern vorzubeugen, muß betont werden, daß diese Steigungsgrad-Karten im mathematischen Sinne nicht exakt sind, zumal da die Steigungsgrade zwischen Isohypsen relativ kleinerer Höhendifferenz bestimmt werden müßten. Von eventuellen Ungenauigkeiten der topographischen Karten muß dabei überhaupt abgesehen werden. Für unsere Versuche darf aber auf den Maßstab der Karte, in der die Darstellung der Reliefenergie

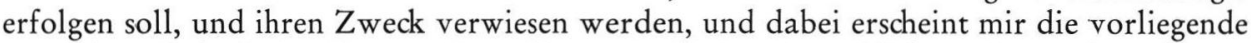
gewählte Methode der Karten-Herstellung als die im Augenblick am besten brauchbare. In puncto Genauigkeit braucht sie einen Vergleich mit den anderen Karten nicht zu scheuen.

1) Für wertvolle Hilfe bei der Herstellung der Karten danke ich den Herren Geoing. MeNgeling, Geoing. Brandes, Dipl.-Geol. Starke, cand. geol. BrüGgemann, Niedersächsisches Landesamt für Bodenforschung, Hannover. 
2.

Der Weg zur Beantwortung der in der Überschrift festgelegten Frage ist mit einer ganzen Reihe von Präjudikaten gepflastert. Zunächst muß man sich darüber im klaren sein, daß entlang einer Eisrandlage ganz unterschiedlich aufgebaute Eisabsätze entstehen. Wir kennen eine Fülle von Beispielen dafür, daß es in weiten Eisrandlagen-Gebieten überhaupt nicht zur Bildung von Endmoränen kam. Und schließlich ist Endmoräne nicht gleich Endmoräne. Eine Stauchendmoräne besitzt eine ganz andere Morphologie als eine Satzendmoräne, und selbst an den Stellen des Eisrandes, an denen Aufschüttungsmoränen entstanden, ging das Ausapern in ganz unterschiedlicher Weise vor sich.

Eisnachschub, Lage der Gletschertore, Gefälle unter und vor dem Eis, Eismächtigkeit, Spaltenbildung, Vorhandensein oder Fehlen von Stauseen vor dem Eise und viele andere Gegebenheiten beeinflussen die Form einer Endmoräne in beträchtlichem Maße. Hinzu kommt das Schicksal der Moräne nach der Aufstauchung oder Aufschüttung. Es ist schließlich nicht unwesentlich, ob eine Endmoräne an ein Gebiet, von dem aus jüngere Erosion sich fortfraß, hydrologisch besonders gut oder schlecht angeschlossen ist. Auch ist zu berücksichtigen, daß Überdeckung durch jüngere Periglazialsedimente, wie z. B. durch Fließerden und Löß, die Form der Moräne sekundär beeinflussen können.

Hat es angesichts dieser Schwierigkeiten überhaupt Sinn, Betrachtungen über die Reliefenergie als Maß für das Alter von Endmoränen anzustellen? Ich glaube, hier gilt der Satz, daß probieren über studieren geht. Wenn man eine gewisse Streuung der Werte in Kauf nimmt, wird man entweder versuchen können, Gesetzmäßigkeiten wahrzunehmen, oder man muß sich in Zukunft hüten zu äußern, eine Moräne sei jung, weil sie "frisch“ aussehe usf.

Als der Verfasser diese Aufgabe in Angriff nahm, rechnete er von vornherein mit einer gewissen Streuung von $\triangle E r$, der durchschnittlichen Reliefenergie gleichaltriger Moränen, nicht nur wegen der primären Unterschiede längs der \pm gleichaltrigen Eisränder, sondern auch weil die Räume, in denen gleichaltrige Moränen entstanden, später nicht selten ein ganz unterschiedliches Schicksal in bezug auf Sedimentation oder Erosion, vor allem aber auf das Klima durchgemacht haben. Eine Weichselmoräne in Schleswig ist schließlich von einer ganz anderen Klimaentwicklung betroffen worden als eine gleichalte Moräne in Masuren.

Versuchen wir trotz aller Einwände, uns nicht von vornherein von der Idee abhalten zu lassen und sehen wir, zu welchen Ergebnissen die Reliefenergiekarten geführt haben!

\section{3.}

Zur Auswahl der Untersuchungsgebiete sind noch einige Bemerkungen notwendig. Nicht alle auf Reliefenergiekarten dargestellten Gebiete sind dem Verfasser ausreichend bekannt, so daß er in der Frage, ob alle darin enthaltenen Areale wirklich Endmoränen sind, weitgehend auf die Auffassung der Kollegen angewiesen ist, die diese Gebiete aufgenommen haben. Bei der Begrenzung der Endmoränen ist nicht den auf den Karten angegebenen, oft verschlungenen geologischen Grenzen genau nachgegangen, sondern diese sind so begradigt worden, daß das dargestellte Gebiet ein geschlossenes Ganzes bildet und die Umgrenzungslinie in etwa die Außenränder der Endmoränenbildungen verbindet. Die gewählten Gesamtgebiete, in denen $\triangle E r$ bestimmt wurden, sind dabei ungefähr gleichgroß und überschreiten nicht die Fläche einer top. Karte 1:25000. Sicher ist, daß, wenn man kleinere Testflächen auswählen würde, eine breitere Streuung der Durchschnittswerte konstatiert werden müßte.

Eine große Anzahl der benutzten Karten ist noch nicht publiziert worden. In diesen Fällen ist auf das Archivmaterial des Niedersächsischen Landesamtes für Bodenforschung zurückgegriffen worden. Den in der in Tab. 1 wiedergegebenen Aufstellung genannten Kollegen ist für ihre Vorarbeiten, dem Niedersächsischen Landesamt für Bodenforschung für die Benutzung der Unterlagen zu danken. 
Tabelle 1

Blatt der GK $25 \quad \triangle \mathrm{Er} \quad$ verwendete Unterlage

\begin{tabular}{|c|c|c|c|}
\hline 1122 & Flensburg & $4,68 \%$ & nach DüCKERs (1958) Übersichtskarte \\
\hline 1222 & Flensburg (Süd) & $2,97 \%$ & nach DüCKERs (1958) Übersichtskarte \\
\hline 1423 & Schleswig & $4,36 \%$ & WOLFF \& HECK (1942) \\
\hline 1726 & Gr. Flintbek & $4,75 \%$ & nach DüCKERs (1958) Übersichtskarte \\
\hline 1729 & Lütjenburg & $5,53 \%$ & nach Übersichtskarte DüCKER 1958 \\
\hline 1730 & Hansühn & $4,15 \%$ & nach Übersichtskarte DüCKER 1958 \\
\hline 1929 & Ahrensbök & $3,14 \%$ & z. T. n. DüCKER 1958 \\
\hline 2128 & Bad Oldesloe & $4,56 \%$ & $\begin{array}{l}\text { RANGE \& SCHLUNCK (1935) } \\
\text { n. d. Auffassung von DüCKER } 1958 \\
\text { (Planungsatlas Schleswig-Holstein) }\end{array}$ \\
\hline 2227 & Bargteheide & $1,81 \%$ & unkartiert, nach Übersichtskarte DüCKER 1958 \\
\hline 2320 & Lamstedt & $3,90 \%$ & SCHRÖDER, H. 1900 \\
\hline 2328 & Trittau & $3,60 \%$ & P. RANGE, 1935 \\
\hline 2330 & Mölln & $4,97 \%$ & GAGEL, C., 1914 \\
\hline 2331 & Seedorf & $4,72 \%$ & BÄrtLing, R. \& GAGEL, C., 1907 \\
\hline 2332 & Groß Salitz & $3,43 \%$ & n. d. Bentz-Karte 1951 \\
\hline 2333 & Groß Brütz & $2,37 \%$ & Bentz \& Mitarb. 1951 (Karte $1: 300$ 000) \\
\hline 2340 & Serrahn & $4,88 \%$ & n. d. WoldstedTschen Karte (1935) \\
\hline 2540 & Malchow & $4,46 \%$ & WOLDSTEDT 1935 \\
\hline 2647 & Fürstenwerder & $3,76 \%$ & F. WAHNSCHAFFE 1893 \\
\hline 2726 & Garlstorf & $3,52 \%$ & [K. Richter 1949] \\
\hline 2742 & Mirow & $4,44 \%$ & GaGEL, C. 1917 \\
\hline 2826 & Evendorf & $3,55 \%$ & [K. RiChTER 1949] \\
\hline 2827 & Amelinghausen & $3,02 \%$ & [K. Richter \& BoigK 1949] \\
\hline 2858 & Arnswalde & $3,55 \%$ & Klautsch, A. \& Behr, J. 1933 \\
\hline 3049 & Gr. Ziethen & $3,48 \%$ & H. SCHROEDER 1896 \\
\hline 3050 & Stolpe & $4,54 \%$ & H. SCHROEDER 1894 \\
\hline 3124 & Falligbostel & $3,38 \%$ & [Boigk \& Niedermayer 1950] \\
\hline 3125 & $\begin{array}{l}\text { Bergen (Westteil) } \\
\text { (Falkenberg-Moräne) }\end{array}$ & $3,91 \%$ & Bentz \& Mitarb. 1951 \\
\hline 3125 & $\begin{array}{l}\text { Bergen (Ostteil) } \\
\text { (Moränen östl. d. Falken- } \\
\text { berg-Moräne) }\end{array}$ & $1,78 \%$ & Bentz \& Mitarb. 1951 \\
\hline 3127 & Unterlüß & $1,88 \%$ & STOLLER, J. 1909 \\
\hline 3128 & Suderburg & $2,23 \%$ & [Niedermayer 1953] \\
\hline 3130 & Bodenteich & $3,69 \%$ & Bentz \& Mitarb. 1951 \\
\hline 3230 & Wittingen & $2,41 \%$ & Bentz \& Mitarb. 1951 \\
\hline 3412 & Fürstenau & $2,58 \%$ & Woldstedt 1935 (Ubersichtskarte) \\
\hline 3414 & Holdorf & $3,24 \%$ & WoLDSTEDT 1935 (Übersichtskarte) \\
\hline 3415 & Damme & $4,71 \%$ & WoLDSTEDT 1935 (Übersichtskarte) \\
\hline 3419 & Nendorf & $2,89 \%$ & WoLDSTEDT 1935 (Ưbersichtskarte) \\
\hline 3422 & Neustadt a. Rbge. & $2,40 \%$ & [HENRICI 1951] \\
\hline 3423 & Otternhagen & $2,21 \%$ & [GENIESER 1963] \\
\hline 3424 & Mellendorf & $2,53 \%$ & [LANG 1963] \\
\hline 3433 & Stolpke & $4,60 \%$ & O. BARSCH 1916 \\
\hline 3437 & Tangermünde & $3,11 \%$ & K. KeIlhack 1902 \\
\hline 3507 & Neuenhaus & $2,80 \%$ & [C. Dietz 1949] \\
\hline 3820 & Rinteln & $7,15 \%$ & NAUMANN, E. 1915 \\
\hline 3822 & Hameln & $7,50 \%$ & NaumanN, E. \& Burre, O. 1922 \\
\hline 3840 & Görzke & $4,23 \%$ & Keilhack, K. \& Schmierer, H. 1904 \\
\hline 3841 & Belzig & $3,69 \%$ & K. KeILHaCK 1904 \\
\hline 4025 & Freden & $9,13 \%$ & LÜTTIG 1952 \\
\hline
\end{tabular}


4.

Vorerst soll Nordwestdeutschland einer Betrachtung unterzogen werden. Die Übersichtskarte (Abb. 1) läßt die Ausschnitte der Endmoränengebiete erkennen, für die Reliefenergiekarten entworfen worden sind. Es ist unschwer festzustellen, daß es sich dabei um winzige Ausschnitte des Gesamtgebietes handelt. Es sollte aber nicht Aufgabe dieser Studie sein, die Frage erschöpfend zu behandeln, sondern es sollte versucht werden festzustellen, ob die angestellten Überlegungen überhaupt Aussicht auf Erfolg und Aussagekraft besitzen.

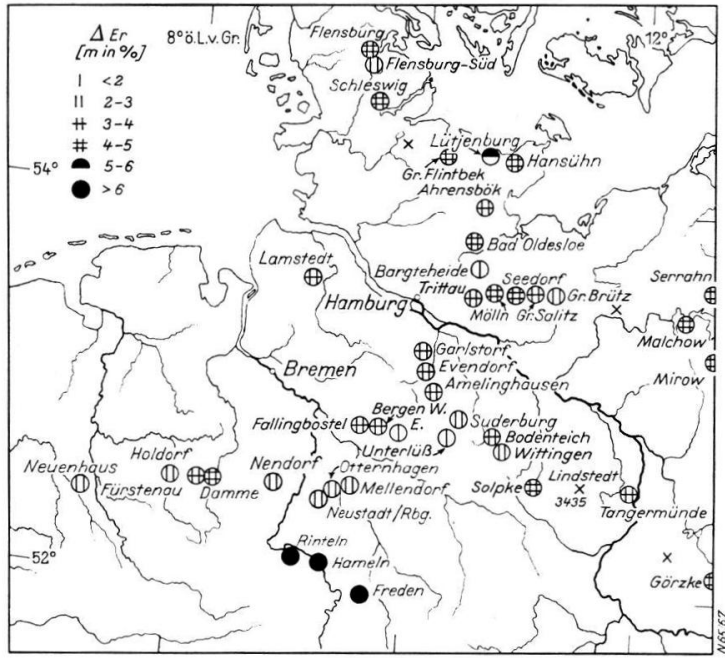

Abb. 1. Ubersicht über Reliefenergiekarten aus Endmoränengebieten NW-Deutschlands.

5.

In Tabelle 1 sind die gemessenen Werte von $\triangle E r$ enthalten. $\mathrm{D}$ a ra us is t $\mathrm{fol}$ gendes ersichtlich: Die in der äußersten und in inneren Eisrandlagen der Weichsel-Eiszeit gelegenen Gebiete weisen eine relativ hohe durchschnittliche Steigung (Durchschnittswert für die ausgewählten Gebiete $=4,01 \%$ ) auf. Die für die einzelnen Testflächen bestimmten Werte sind aber keinesfalls einheitlich. Bereits bei aneinandergrenzenden Blättern sind, wie der Fall Flensburg und Flensburg-Süd zeigt, größere Unterschiede $(4,68 \%$ zu 2,97\% \%) bemerkbar. Im genannten Beispiel ist die Differenz auf den unterschiedlichen Charakter (im einen Falle liegen vorwiegend nahe dem ehemaligen Gletschertrog und der glaziären Übertiefung gelegene Gebiete, im anderen distale Teile der Endmoränen vor) und die morphologische Situation innerhalb des Moränen-Komplexes zurückzuführen.

Im Falle des Gebietes Bargteheide handelt es sich um Moränenteile in einem schlecht ernährten Eisrandteil abseits der Haupt-Ausaperungszonen.

Daraus geht hervor, daß man eigentlich nur Endmoränen, die in gleicher eismorphologischer Situation gelegen haben, miteinander vergleichen sollte. In vielen Fällen weiß man aber, bevor man die Reliefenergie-Karte entwirft, derartiges nicht, sondern wird erst durch die Reliefenergie-Karte auf Absonderheiten aufmerksam; ein Grund mehr, ihre Konstruktion zu empfehlen.

Insgesamt gesehen bestätigt sich diese bereits in der Bezeichnung „Jungmoränen “ unterschwellig liegende Klassifikation, die an eine relativ bewegte Morphologie denken läßt. Generell ist auch beobachtbar, daß die weiter östlich gelegenen Testfelder im allgemeinen eine geringere durchschnittliche Reliefenergie besitzen, was sowohl mit primär weniger lebhafter Ausaperung als auch sekundär mit geringerer posthumer Erosion und anderen 
Ursachen zusammenhängen kann. Die W a r the - M o rän e n, die aus einer Reihe vorwiegend morphostratigraphischer Gründe (z. B. Fehlen offener Seen, stärkerer Verschleifung durch periglaziale Ereignisse, stärkerer Verwitterung, geringerer „Frische“) mit den Drenthe-Moränen zum stratigraphischen Komplex der Saale-Eiszeit vereinigt worden sind, weisen in der Tat eine durchschnittlich geringere Reliefenergie auf ( $\triangle E r$ aller vermessener Gebiete $=3,27 \%$ ). Dort, wo besonders niedrige durchschnittliche Steigungsgrade gemessen worden sind, handelt es sich um Vertretung der Endmoränen durch Sanderwurzeln bzw. Anschoppungsgebiete des Wartheeises an Drenthe-Eisrandsedimente.

Von den Drenthe-Moränen sind zwei Gruppen betrachtet worden. Die eine umfaßt den Bereich der Rehburger bis Heisterberg-Phase westlich von Hannover. Hier betrug $\triangle E r$ von allen Testgebieten 2,97\%. Auch das entspricht den bisherigen Ansichten. Abweichungen vom Durchschnitt (z.B. zwischen Bl. Holdorf und Bl. Damme) sind zwanglos durch die unterschiedlich starke Stauchung im Endmoränenbereich erklärbar.

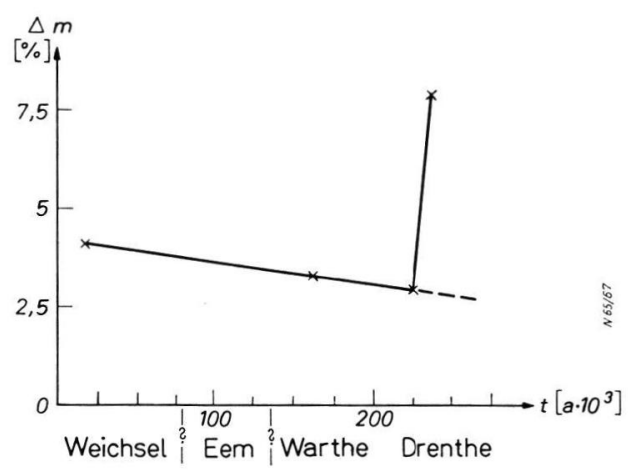

Abb. 2.

Nach dem bisher Gesagten würde man daher, falls man zu dem kühnen Schritt eines Diagrammes Reliefenergie : Alter der Moränen gehen wollte, erwarten, daß die Kurve $\triangle E r: t$ in Richtung auf das höhere Alter weiterhin abfällt. Abb. 2 zeigt aber einen bemerkenswerten Knick in der Kurve. $\triangle E r$ ist hier wesentlich höher.

Dieser Befund kann sehr leicht erklärt werden, und zwar wie folgt:

1) Bei den Testgebieten, die allesamt im Gebirgslande liegen, handelt es sich um viel kleinere Areale als bei den Endmoränen-Gebieten im Flachlande, bei denen es oft zur Sache des Taktes wird, wie weit man die Umgrenzung der Endmoränen zieht. Bei den Gebirgsland-Gebieten handelt es sich mit Sicherheit um Absätze unmittelbar am Eisrande, im Flachland läßt sich dieser schwieriger rekonstruieren, weshalb auch eisrandfernere Gebiete mit im Testgebiet enthalten sein können.

2) Die Endmoränen im Gebirgslande sind durch kleine Talgletscher aufgeschüttet bzw. aufgestaucht worden. Das subglaziäre und extraglaziäre Relief war sehr lebhaft. Die Sedimentation in diesem stark reliefierten Gebiet erfolgte unter viel bewegteren Begleiterscheinungen als im Flachlande.

3) Die postglaziale Erosion war im Gebirgslande viel lebhafter als im Flachlande.

Mithin können diese Moränen in bezug auf die in der Überschrift des Aufsatzes gestellte Frage nicht mit den Flachlandmoränen verglichen werden.

6.

Wie sich herausstellt, ist neben der primären auch die postglaziale (wobei unter postglazial der Zeitraum nach der betr. Vereisung gemeint ist, also im Falle Drenthe der ge s a m t e Zeitraum post Drenthe-Glazial) Formung von Einfluß auf die Reliefenergie 
der Moränen. Sicherlich kann erwartet werden, daß Unterschiede des Klimas auch eine unterschiedliche Formung gleichalter Moränen zur Folge haben.

Aus diesem Grunde sollten durch spätere Weiterführung der Betrachtung ehemaliger Eisränder, z. B. die von Warthe und Weichsel, weiter nach Osten verfolgt werden.

Diese Arbeit konnte aus Zeitgründen vorerst nicht abgeschlossen werden. Da der Inhalt des vorliegenden Artikels in seinen wesentlichen Punkten aber bereits durch Vorträge bekannt geworden ist, soll die Publikation der grundsätzlichen Erkenntnisse nicht länger hinausgezögert werden. Durch zusätzliche Messungen in weiteren Endmoränengebieten ist beabsichtigt, nicht nur der Frage des durch unterschiedliches Klima bedingten sekundären Formenwandels, sondern auch anderen Problemen, wie der spezifischen morphologischen Eigenart der verschiedenen Moränentypen, der Beziehung zwischen Großrelief und Kleinrelief sowie den Zusammenhängen zwischen Gewässernetzdichte und Oberflächenform der Moränen nachzugehen.

\section{Literatur}

Bärtling, R. \& Gagel, C.: Geologische Karte von Preußen etc., 1 : 25000, Blatt Seedorf. Berlin 1907.

BarsCh, O.: Geologische Karte von Preußen etc., 1 : 25 000, Blatt Solpke. Berlin 1916.

Benrens, Sven E.: Morphometrische, morphogenetische und tektonische Studien der nordwestschonischen Urgebirgsrücken, mit besonderer Berücksichtigung von Kullaberg. - Lund Studies in Geography, Ser. A., No. 5, Lund 1953.

Bentz, A. \& Mitarb.: Geologische Übersichtskarte von Nordwestdeutschland 1:300000. Hannover 1951.

DüCKeR, A.: Geologische Karte von Schleswig-Holstein. In: Deutscher Planungsatlas. Kiel 1958.

Gagel, C.: Geologische Karte von Preußen etc., $1: 25$ 000, Blatt Mölln. Berlin 1914. - - Geologische Karte von Preußen etc., 1 :25 000, Blatt Mirow. Berlin 1917.

Kerlhack, K.: Geologische Karte von Preußen etc., 1:25 000, Blatt Tangermünde. Berlin 1902. - - Geologische Karte von Preußen etc., 1:25 000, Blatt Belzig. Berlin 1904.

Keilhack, K. \& Schmierer, Th.: Geologische Karte von Preußen etc., $1: 25$ 000, Blatt Görzke. Berlin 1904.

Klautsch, A. \& Behr, J.: Geologische Karte von Preußen etc., 1 : 25 000, Blatt Arnswalde. Berlin 1933.

LütтіG, G.: Alt- und mittelpleistozäne Eisrandlagen zwischen Harz und Weser. Inaug. Diss., Göttingen 1952. Geol. Jb. 70, 43-125, Hannover 12. 1954. - - Eisrand und Reliefenergie. - N.Jb. Paläont. Mh. 1953, 1, 16-20, Stuttgart 1953. - - Hat sich der Nordwestharz im Postglazial gehoben? Geol. Jb. 70, 405-434, 11 Abb., Hannover 1955.

Naumann, E.: Geologische Karte von Preußen etc., $1: 25$ 000, Blatt Rinteln. Berlin 1915.

Naumann, E. \& Burre, O.: Geologische Karte von Preußen etc., 1 : 25000 , Blatt Hameln. Berlin 1922.

Range, P.: Geologische Karte von Preußen etc., Blatt Trittau. Berlin 1935.

Range, P. \& Schlunk, J.: Geologische Karte von Preußen etc., $1: 25000$, Blatt Bad Oldesloe. Berlin 1935.

Schroeder, H.: Geologische Karte von Preußen etc., $1: 25000$, Blatt Stolpe. Berlin 1894. - Geologische Karte von Preußen ety., 1:25000, Blatt Gr. Ziethen. Berlin 1896. - Geologische Karte von Preußen etc., 1: 25 000, Blatt Lamstedt. Berlin 1900.

Stollek, J.: Geologische Karte von Preußen etc., 1 : 25 000, Blatt Unterlüß. Berlin 1909.

Thauer, W.: Neue Methoden der Berechnung und Darstellung der Reliefenergie. Peterm. Mitt. 1955, 8-13, Gotha 1955.

Wahnschaffe, F.: Geologische Karte von Preußen etc., $1: 25000$, Blatt Fürstenwerder. Berlin 1893.

Waldbaur, H.: Die Reliefenergie in der morphologischen Karte. Peterm. geogr. Mitt. 96, 3, 156167 , Gotha 1952.

Woldstedt, P.: Geologisch-morphologische Karte des norddeutschen Vereisungsgebietes 1:500000. Berlin (Preuß. Geol. L.-A.) 1935.

WolfF, W. \& Heck, H.-L.: Blatt Schleswig. Geologische Karte von Deutschland, $1: 25$ 000. Berlin 1942.

Manuskr. eingeg. 22.6. 1968.

Anschrift des Verf.: Dr. G. Lüttig, Ltd. Regierungsdirektor im Niedersächsischen Landesamt für Bodenforschung, 3 Hannover-Buchholz, Alfred-Bentz-Haus. 